\title{
Multi-satellite Observation Scheduling for Large Area Disaster Emergency Response
}

\author{
X. N. Niu ${ }^{1,2}$, H. Tang ${ }^{1,2, *}$, L. X. Wu ${ }^{3}$ \\ ${ }^{1}$ Key Laboratory of Environment Change and Natural Disaster, Ministry of Education, Beijing Normal University, Beijing, \\ 100875, China - niuxiaonan@mail.bnu.edu.cn, hongtang@bnu.edu.cn \\ ${ }^{2}$ Beijing Key Laboratory of Environmental Remote Sensing and Digital Cities, Beijing Normal University, Beijing, 100875, \\ China \\ ${ }^{3}$ School of Geosciences and Info-Physics, Central South University, Changsha, 410083, China - awulixin@263.net
}

\section{Commission III, ICWG III/IVa}

KEY WORDS: Disaster Emergency Response, Area Tasks, Decomposition, Multi-satellite Scheduling, Multi-objective Genetic Algorithm

\begin{abstract}
:
Timely acquiring remote sensing data is very important for rapid response to disasters. Satellite task scheduling aiming at making an optimal imaging plan, plays a key role in coordinating multiple satellites to monitor the disaster area. In the paper, to generate imaging plan dynamically according to the disaster relief, we propose a dynamic satellite task scheduling method for large area disaster response. First, an initial robust scheduling scheme is generated by a robust satellite scheduling model in which both the profit and the robustness of the schedule are simultaneously maximized. Then, we use a multi-objective optimization model to obtain a series of decomposing schemes. Based on the initial imaging plan, we propose a mixed optimizing algorithm named HA_NSGA-II to allocate the decomposing results thus to obtain an adjusted imaging schedule. A real disaster scenario, i.e., 2008 Wenchuan earthquake, is revisited in terms of rapid response using satellite resources and used to evaluate the performance of the proposed method with state-of-the-art approaches. We conclude that our satellite scheduling model can optimize the usage of satellite resources so as to obtain images in disaster response in a more timely and efficient manner.
\end{abstract}

\section{INTRODUCTION}

\subsection{General Instructions}

For disaster emergency response, the most critical information during the three days immediately following a disaster event are

the accurate and timely intelligence about the extent, scope and impact of the event (Liu and Hodgson, 2016). Based on the information, disaster management department and decision-makers can make advisable relief strategies. The earth observation satellite can be used to perform a wide range of observation activity to obtain the information of disaster formation, development and dynamic change, which can provide data of disaster monitoring timely.

Although much work has been done on information extraction for disaster reduction from satellite images, little attention has been paid to how to efficiently schedule multiple earth observation satellites to make an optimal imaging plan to meet requirements for disaster response. For remote sensing applications during the response phase, the first practical problem is satellite task scheduling. The scheduling can be primarily divided into static scheduling and dynamic scheduling. The static scheduling assumes that all imaging tasks have been submitted before scheduling, and once the scheduling scheme is produced, it is immutable until all tasks have been finished. Because natural disasters (earthquakes, landslides, debris flow, etc.) often happen unexpectedly, it is suggested to use dynamic scheduling methods to cope with these unexpected factors.
Dynamic scheduling has been defined under three categories (Ouelhadj and Petrovic, 2009): completely reactive scheduling, predictive-reactive scheduling, robust predictive-reactive scheduling and robust pro-active scheduling.

In completely reactive scheduling, all imaging tasks are dispatching in real-time, thus no initial schedule is generated in advance. Priority dispatching rules are frequently used. The literature (Qiu et al., 2013) proposed a rolling horizon strategy to deal with new arriving tasks. They designed various heuristic algorithms to schedule tasks. The heuristic algorithms are quick and easy to implement. However, the decisions are made locally and it is hard to predict system performance.

Predictive-reactive scheduling is the most common dynamic scheduling method used in satellite scheduling. An initial imaging plan is produced in advance and when emergent events occur unexpectedly, the initial scheme will be revised. Literature (Pemberton and Greenwald, 2002) developed the satellite scheduling problem and discussed contingency conditions under which the satellite scheduling problem becomes dynamic. The literature (Verfaillie et al., 1994) proposed an approach for reusing any previous schedule employing local adjustment. The literature (Sun et al., 2010) presented dynamic scheduling problem as a dynamic weighted maximal Constraint Satisfaction Problem and adopt genetic algorithm to obtain a satisfactory solution. In predictivereactive scheduling, the new schedule may have large difference with the initial solution, which can seriously affect

* Corresponding author 
other observing tasks in the initial schedule and may lead to poor performance of the schedule.

Robust predictive-reactive scheduling aims at developing predictive-reactive schedules to minimize the perturbation to the previous schedule. Considering the tradeoff between the performance and the degree of the adjustment, the literature (Wang et al., 2007) proposed a robust scheduling model and adopted a rule-based heuristic algorithm to solve the dynamic scheduling problem.

The literatures (Niu et al., 2015, Zhai et al., 2015) propose a robust multi-objective scheduling model to generate robust initial solutions so as to minimize the difference between the adjusted schedule and the initial schedule.

Robust pro-active scheduling strategy focus on obtaining predictive schedules which satisfy performance requirements predictably in a dynamic environment (Ouelhadj and Petrovic, 2009). However, the environment is very difficult to predict and the determination of the predictability measures is also hard.

To sum up, the robust predictive-reactive scheduling approach is appropriate to generate stable imaging plans for response to practical emergent tasks. During the disaster relief process, the disaster information obtained changes dynamically, which accordingly leads to new imaging requirement. In the paper, we focus on the dynamic emergent imaging requirements. A robust multi-satellite dynamic scheduling model is proposed to address the area emergent tasks. A multi-objective genetic algorithm is used to decompose the area task into a series of adjacent strips. Then we present the multi-objective genetic algorithm embedding in a heuristic rule to obtain the dynamic scheduling plans.

The remainder of the paper is organized as follows. In section 2 , we describe the dynamic scheduling model. Then we present the satellite scheduling of area tasks algorithm in section 3. In section 4, we conduct experimental simulations. We conclude the paper with a summary in section 5 .

\section{DYNAMIC SCHEUDLING MODEL}

To deal with the new emergent tasks, we use a robust predictreactive scheduling strategy. Based on a robust initial schedule obtained by a robust scheduling model (Zhai et al., 2015), we build a dynamic satellite scheduling model to adjust the current imaging plan so as to arrange the new coming emergent tasks. The model includes five parts.

\subsection{Tasks}

The paper mainly focuses on emergent area tasks which cannot be image in one shot. The area task must be decomposed into several strips and each strip is allocated to a satellite within a time window. Considering the disaster events usually occur unexpectedly, with uncertainties of occurrence time and number. So the emergency task number and arrival times are not known a priori. Moreover, the emergent tasks need to be completed in time, because rapid information acquisition during the three days after a disaster event is crucial.

Let $T^{E}=\left\{T^{E_{1}}, T^{E_{2}}, \cdots, T^{E_{n}}\right\}$ be the emergency area task set. $T^{E_{i}}$ denotes the emergent task for the $i$-th phase. Each task is associated with the weight $p_{i}$, the indispensable duration of task execution $d_{i}$.

Considering that many tasks are commonly submitted together, thus we assume in this paper the new tasks arrive in batch style. Let $T S=\left\{T_{S}^{1}, T_{S}^{2}, \cdots, T_{S}^{n}\right\}$ be the dynamic scheduling time set where $T_{S}^{i}$ is the $i$-th dynamic scheduling time.

\subsection{Satellites}

Multiple satellites is used to acquire images of these tasks, which are denoted as $S=\left\{s^{1}, \cdots s^{J}\right\}$. For each satellite $s^{j} \in S$, its key parameters used in the model include the field of view $\Delta \theta^{j}$, the longest duration allowed for a continuous observation $\Delta d^{j}$, slewing rate, $s l^{j}$, attitude stability time $s t^{j}$, the maximum swing angle $m s g^{j}$, the flight time in each orbit $o r b^{j}$, the start-up time of sensor $s u^{j}$, the retention time of shutdown $s d^{j}$, and the longest imaging time $d u t y^{j}$ in each orbit, respectively.

\subsection{Time windows}

Satellites' observing activities must performed within the available time windows between tasks and satellites. For a task $t_{i}$, its corresponding the time window is $t w_{\mathrm{i}}^{j}=\left[w s_{i}^{j}, w e_{i}^{j}\right]$ with the observation angle $\theta_{i}^{j}$. We define the time windows of $t_{i}$ as a set $T W_{i}=\bigcup_{j=1}^{J} \bigcup_{k=1}^{K_{i j}} t w_{i k}^{j}, K_{i j}$ is the number of time windows between task $t_{i}$ and satellite $s^{j}$.

\subsection{Objectives}

The model is to produce an imaging plan to maximize the revenue of the emergent tasks as well as to minimize the difference between the new schedule and the initial schedule. So the model include two objectives: accumulated revenue of the allocated emergency tasks and the disturbance of the schedule made by the previous phase. We assume that $T_{S}^{k}$ is the current scheduling time.

In the model, all the emergent tasks have large scope and a satellite often cannot acquire the entire area in a single pass. The area targets must be partitioned into a set of contiguous strips based on the different characteristics of the satellites and disaster emergent imaging requirements. Therefore, before satellite scheduling, we use a multi-objective optimizing model (Niu et al., 2018) to segment the emergent area tasks so as to obtaining a group of decomposing results, which are the inputs of the scheduling model.

The first objective is to maximize the profit of the observed area of emergency tasks.

$$
\max : P R O\left(S S^{k}\right)=\sum_{i=1}^{\left|T_{k}^{E}\right|} \max \left(\mathrm{U}_{m=1}^{M_{i}} \operatorname{COV}(m) \times p_{i}\right)
$$

where $M_{i}$ denotes the number of decomposing results of the area task $i$ and $\operatorname{COV}(\mathrm{m})$ is the proportion of area of the target covered by all selected strips of the decomposing solution $m$. 
The second objective is to minimize the difference between the adjusted schedule $S S^{k}$ and the initial schedule $S S^{k-1}$. Generally, the scheduled emergent tasks may have two types of variances in dynamic scheduling: (1) change of finish time and (2) rejection (Wang et al., 2015).

$$
\min : \operatorname{PER}\left(S S^{k}\right)=\sum_{i=1}^{\left|S S^{k 1}\right|} \sum_{q=1}^{2} \operatorname{disturb}(i, q) \times w_{q}
$$

where $\operatorname{disturb}(i, q)$ is the count of variance 1 on task $t_{i}$ in the whole scheduling. $\omega_{q}, q=1,2$ represents the influence degree of type $q$ variance, and generally $\omega_{1}<\omega_{2}$.

\subsection{Constraints}

In our model, regardless of the satellite measurement and control requirements and the data transmission with ground station requirements, there are four constraints that must be satisfied in the model.

\subsubsection{Time window constraint}

Any meta-tasks must be observed within its time windows:

$$
\left\{\begin{array}{l}
x_{i k v}^{j}\left(t s_{i k v}^{j}-w e_{i k v}^{j}\right) \geq 0 \\
x_{i k v}^{j}\left(t s_{i k v}^{j}+d_{i k v}^{j}-w e_{i k v}^{j}\right) \leq 0 \\
a t_{i} \leq T_{S}^{k} \leq t s_{i k v}^{j} \leq d t_{i}
\end{array}\right.
$$

where $t s_{i k v}^{j}$ presents the start time of the meta-task $r o_{i k v}^{j}$.

\subsubsection{Switch time constraint}

The transition time between any two successive tasks for the same satellite $j$ should be enough for sensor to execute a series activities including shutting down, pointing to the target, stabilizing gesture and start-up.

$$
t e_{m}^{j}+s d^{j}+t r_{m, n}^{j}+s u^{j} \leq t s_{n}^{j}
$$

where $t e_{m}^{j}$ is the end time of the previous task $t_{m}, t r_{m, n}^{j}$ denotes the transition time between task $t_{m}$ and the next task $t_{n}$.

\subsubsection{Imaging time constraint}

The total imaging time of any satellite $s^{j}$ should be less than the allowable longest imaging time for one orbit.

$$
\sum_{i \in S S_{h}^{j}} d_{i} \leq d u t y^{j}
$$

where $S S_{b}^{j}$ denotes a sequence of scheduled tasks on satellite $j$ on orbit $b$.

\subsubsection{Storage constraint}

The storage capacity of a satellite is limited, so only limited number of tasks can be scheduled.

$$
\sum_{l=1}^{L^{j}} d_{l}^{j} \times m \leq M^{j}
$$

where $M^{j}$ is the maximum storage capacity of satellite $j . L^{j}$ is the number of tasks allocated to satellite $j$.

\section{ALGORITHM}

Since the impact area caused by major natural disasters is generally large which cannot be imaged in one shot by a single satellite. Therefore, the area target must be decomposed into several contiguous strips which is called meta-tasks. Considering multiple emergency imaging requirements, we use a multi-objective optimization model to obtain a group of decomposing results. Based on the decomposing results, we use a mixed algorithm HA-NSGA2 to insert the meta-tasks into the current scheduling scheme. The process to solve the model is shown in Figure 1.

As shown in Figure 1, the solving method begins with producing a set of decomposing schemes for the area task using multi-genetic algorithm. This process is to search for the combinations of meta-tasks covering the area target considering multiple emergent imaging requirements such as the extent of coverage over the stricken area, timeliness, and the spatial resolution. Each decomposition result contains a series of specific time windows within which the associated satellite can observe a part of the target area. Based on the partitioning solutions, using the HA_NSGA-II algorithm, we insert all the meta-tasks belonging to a decomposition scheme into the current imaging plan. As a result, a group of adjusted scheduling schemes is obtained. The scheduling scheme with maximum profit and minimum perturbation is selected as the final solution.

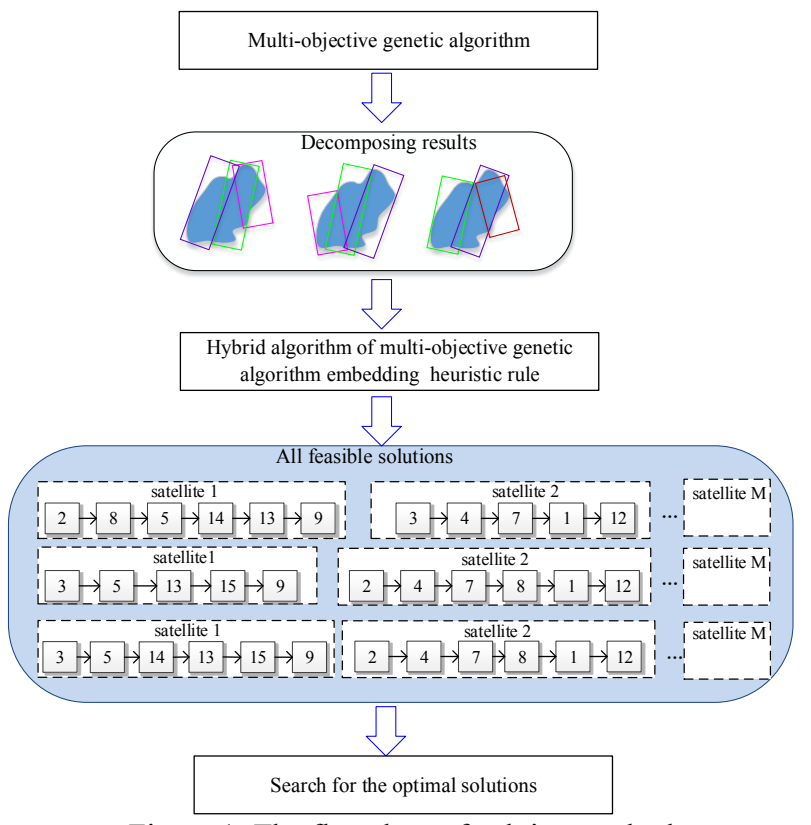

Figure 1. The flowchart of solving method

\section{EXPERIMENT}

We take Wenchuan Earthquake as the emergent event. After the earthquake occur, users will summit the imaging requirements. Based on the situation of disaster relief at that time, we simulate three batches of emergent tasks. As shown in Table 1, we describe the experimental scenarios. The location and scope of the area tasks are presented in Figure 2 the scheduling period is set as three days. 

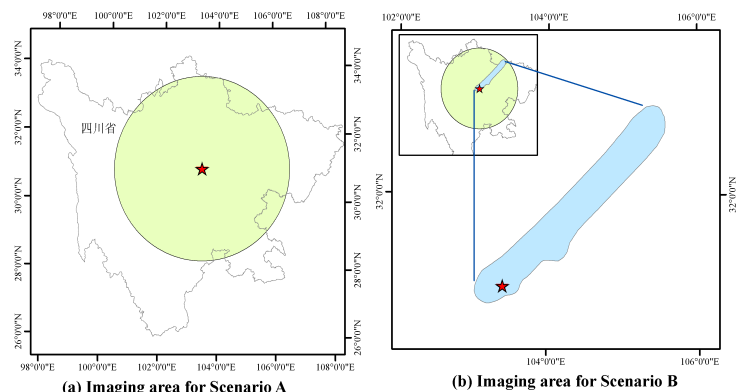

图例 $\star$ Epicenter $\square$ area target $1 \quad \square$ area arret 2

Figure 2. The target area

\begin{tabular}{|c|c|c|c|}
\hline Scenarios & Purpose & Target area & $\begin{array}{c}\text { Imaging } \\
\text { requirements }\end{array}$ \\
\hline $\begin{array}{c}\text { Scenario } \\
\text { A }\end{array}$ & $\begin{array}{l}\text { roughly } \\
\text { grasping the } \\
\text { extent of } \\
\text { damage area }\end{array}$ & $\begin{array}{l}\text { A buffer } \\
\text { zone with } \\
\text { a radius of } \\
300 \mathrm{~km} \\
\text { centered } \\
\text { on the } \\
\text { epicenter }\end{array}$ & $\begin{array}{l}\text { Spatial } \\
\text { resolution: } \\
1-10 \mathrm{~m} .\end{array}$ \\
\hline $\begin{array}{c}\text { Scenario } \\
\text { B }\end{array}$ & $\begin{array}{c}\text { Evaluating } \\
\text { the damage of } \\
\text { the worst-hit } \\
\text { area }\end{array}$ & $\begin{array}{l}\text { Ninth- } \\
\text { degree } \\
\text { seismic } \\
\text { intensity } \\
\text { region }\end{array}$ & $\begin{array}{l}\text { Spatial } \\
\text { resolution: } \\
0-5 \mathrm{~m} .\end{array}$ \\
\hline $\begin{array}{c}\text { Scenario } \\
\mathrm{C}\end{array}$ & $\begin{array}{l}\text { Seeking data } \\
\text { sharing from } \\
\text { international } \\
\text { satellites of } \\
\text { CHARTER }\end{array}$ & $\begin{array}{l}\text { Ninth- } \\
\text { degree } \\
\text { seismic } \\
\text { intensity } \\
\text { region }\end{array}$ & $\begin{array}{l}\text { Spatial } \\
\text { resolution: } \\
0-1 \mathrm{~m} .\end{array}$ \\
\hline
\end{tabular}

Table 1. The information about emergent tasks

When the earthquake breaks out, there is an urgent need to acquire the information about stricken area as soon as possible. The first imaging requirement is to acquire the information to grasp the extent of impact area. We use the HA_NSGA-II algorithm to obtain the imaging plans for this scenario, as shown in Figure 4. It can be found that the area task is decomposed into multiple strips which are assigned to satellites and time windows. The target area is entirely covered by observation strips. In the scenario B, the worst-hit area of earthquake is set as the observing target, namely $T_{2}^{E}$. Hence, the new imaging requirement triggers the second dynamic scheduling. Based on the previous scheduling scheme (solution\#1), we schedule new emergent tasks to and the adjusted scheduling schemes are produced, as depicted in Figure 5. The result indicates that a subset of meta-tasks are adjusted to satisfy new imaging requirement. To obtain veryhigh resolution images, we seek for data sharing from international satellites of CHARTER. The scheduling results are as shown in Figure 6. There are different imaging plans and those solutions with maximum profit and minimum perturbation should be selected as the final imaging plan, as shown in Figure 3.

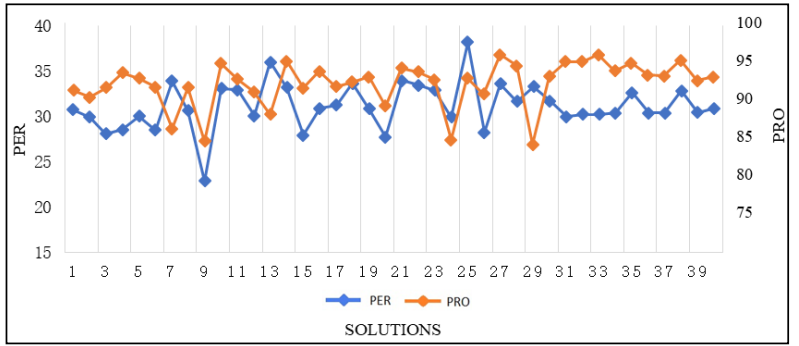

Figure 3. The objectives of the scheduling results

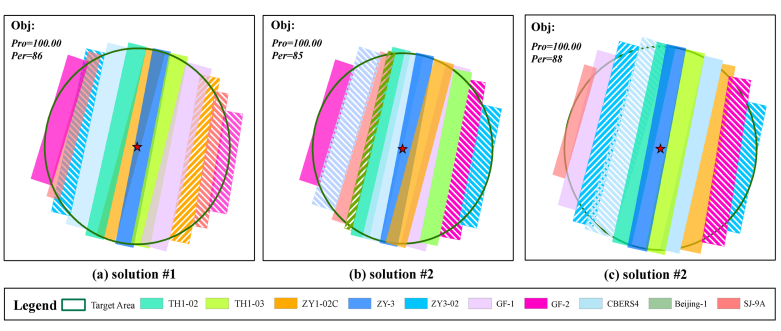

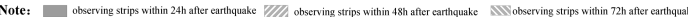

Figure 4. The scheduling schemes for Scenario A

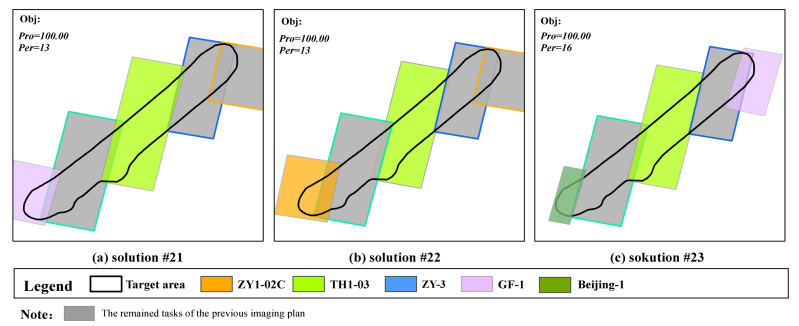

Figure 5. The scheduling schemes for Scenario B
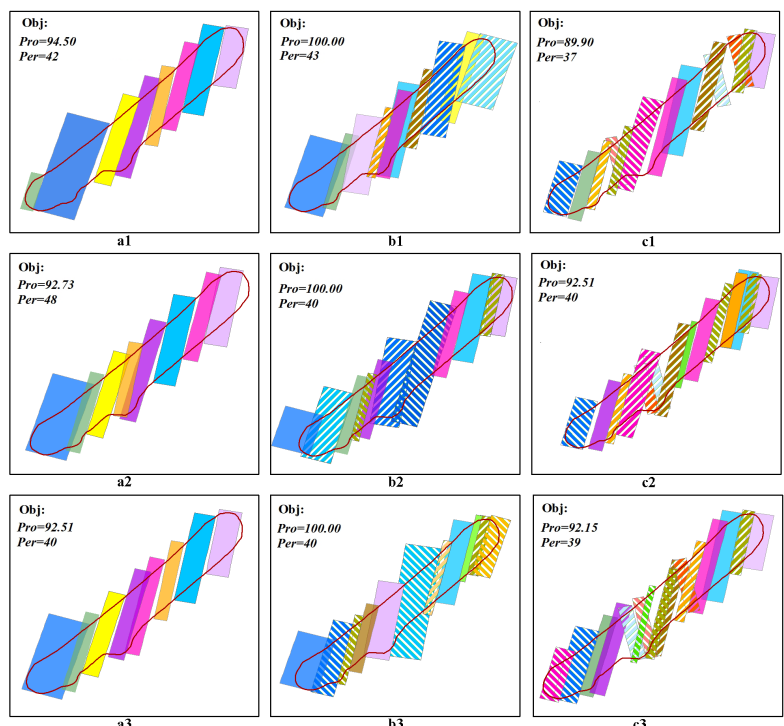

Legend $\square$ Area target $\square$ WorldView-1 $\quad$ WorldView-2 $\quad$ WorldView-3 $\quad$ Ikonos-2 $\quad$ EROS-B

$\square$ Pleiades-1A $\square$ Pleiades-1B $\square$ Cartosat-2 $\square$ Cartosat-2A $\square$ Cartosat-2B VIIIA Geoe Eye-

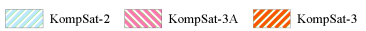

Note: strips observed within the first day of the scheduling period TIII strips observed within the second day of the scheduling period

NV strips observed within the second day of the scheduling period

Figure 6. The scheduling schemes for Scenario C

\section{CONCLOSION}

To address the scheduling problem oriented to the dynamic area tasks triggered by emergent disasters, we build a dynamic scheduling model in which the profit of the emergent tasks is 
maximized and the perturbation to the initial schedule is minimized. The multi-objective genetic algorithm is used to generate decomposing schemes of the area tasks. Then we employ the HA_NSGA-II algorithm to obtain the dynamic scheduling result. To evaluate our model, we conduct experimental simulations in the scene of Wenchuan Earthquake. The simulated imaging plan can schedule satellites to observe a wide scope of target area. We conclude that our satellite scheduling method can deal with the emergent imaging requirements of large area target.

\section{ACKNOWLEDGEMENTS}

This work was supported by the National Key R\&D Program of China (Grant No. 2017YFB0504104), in part by the Foundation Research Funds for the Central Universities and in part by XJCC Innovation Team of Geospatial Information Technology (Grant No. 2016AB021).

\section{REFERENCES}

B. Sun, W. Wang, and Q. Qi, "Satellites scheduling algorithm based on dynamic constraint satisfaction problem," in Computer Science and Software Engineering, 2008 International Conference on, 2008, pp. 167-170.

D. Ouelhadj and S. Petrovic, "A survey of dynamic scheduling in manufacturing systems," Journal of scheduling, vol. 12, pp. 417-431, 2009.

G. Verfaillie and T. Schiex, "Solution reuse in dynamic constraint satisfaction problems," in AAAI, 1994, pp. 307-312.

J. C. Pemberton and L. Greenwald, "On the need for dynamic scheduling of imaging satellites," International Archives of Photogrammetry Remote Sensing and Spatial Information Sciences, vol. 34, pp. 165-171, 2002.

J. Wang, J. Li, and Y. Tan, "Study on heuristic algorithm for dynamic scheduling problem of earth observing satellites," in Software Engineering, Artificial Intelligence, Networking, and Parallel/Distributed Computing, 2007. SNPD 2007. Eighth ACIS International Conference on, 2007, pp. 9-14.

J. Wang, X. Zhu, L. T. Yang, J. Zhu, and M. Ma, "Towards dynamic real-time scheduling for multiple earth observation satellites," Journal of Computer and System Sciences, vol. 81, pp. 110-124, 2015.

Q. Dishan, H. Chuan, L. Jin, and M. Manhao, "A Dynamic Scheduling Method of Earth-Observing Satellites by Employing Rolling Horizon Strategy," The Scientific World Journal, vol. 2013, 2013.

S. Liu and M. E. Hodgson, "Satellite image collection modeling for large area hazard emergency response," ISPRS Journal of Photogrammetry and Remote Sensing, vol. 118, pp. 13-21, 2016.

X. Niu, H. Tang, L. Wu, R. Deng, and X. Zhai, "Imagingduration embedded dynamic scheduling of Earth observation satellites for emergent events," Mathematical Problems in Engineering, vol. 2015, pp. 1-31, 2015.

X. Zhai, X. Niu, H. Tang, L. Wu, and Y. Shen, "Robust Satellite Scheduling Approach for Dynamic Emergency
Tasks," Mathematical Problems in Engineering, vol. 2015, pp.1-20, 2015.

X. Niu, H. Tang, L. Wu, "Satellite scheduling of large area tasks for rapid response to natural disaster reduction using a multi-objective genetic algorithm," International Journal of Disaster Risk Reduction, 2018. (Online). 